\title{
Cytoskeleton-associated antigens from African trypanosomes are recognized by self-reactive antibodies of uninfected mice
}

\author{
N. MÜLLER ${ }^{1}$, M. IMBODEN ${ }^{1}$, E. DETMER ${ }^{1}, J$. M. MANSFIELD ${ }^{2}$ and T. SEEBECK ${ }^{1 *}$ \\ ${ }^{1}$ Institut für Allgemeine Mikrobiologie, University of Bern, Baltzerstrasse 4, CH-3012 Bern, Switzerland \\ 'Department of Animal Health and Biomedical Sciences, 1655 Linden Drive, University of Wisconsin, Madison, \\ WI 53706, USA
}

(Received 4 November 1992 ; revised 15 March 1993; accepted 22 March 1993)

\section{SLMMARY}

Serum from uninfected mice of different strains, as well as from germ-free animals, contains antibodies which react specifically with at least two trypanosomal proteins, I/6 and MARP1. These antibody populations are highly specific for the respective proteins, are of similar affinity as hyperimmune antibodies, and consist of $\operatorname{IgM}$ as well as IgG isotypes. Hyperimmune antibody raised against the cross-reacting trypanosomal protein $\mathrm{I} / 6$ detects a $60 \mathrm{kDa}$ protein in mouse $3 \mathrm{~T} 6$ cells, which is a component of the fibroblast cytoskeleton.

Key words: cytoskeletal-associated antigens, African trypanosomes, antibody response.

\section{INTRODUCTION}

Mammalian hosts which have been infected with African trypanosomes undergo a strong, polyclonal B-cell stimulation and a concomitant increase in antibody production (Bancroft \& Askonas, 1985; Morrison, Murray \& Akol, 1985; Shapiro \& Pearson, 1986). This stimulation is followed, at a later stage of the infection, by significant immunosuppression, which may be induced by the appearance of lymphocytes bearing anti-idiotypic receptors (Theodos \& Mansfield, 1990). While the major part of the immune response mounted after infection is directed against the variable surface glycoproteins (VSGs; Cross, 1990), antibodies against other parasite proteins are also detected (Shapiro \& Murray, 1982; Burgess \& Jerrells, 1985; Müller et al. 1992).

Current efforts in our laboratories are devoted to analysing the early stages of trypanosome infections. Two non-variant antigens, to which antibodies are detectable very early after infection, have recently been characterized at the molecular level (Hemphill, Affolter \& Seebeck, 1992; Müller et al. 1992). One of these, MARP1, is a microtubule-associated protein which, upon cell fractionation, is found in the heatresistant high molecular weight fraction of trypanosomes. The other of these proteins, GM6, is located at the cell-body/flagellum interface.

The present study analyses the time-course of the early antibody response to non-variant trypanosome antigens in mice. It demonstrates that an antibody

* Reprint requests to: Dr T. Seebeck, Institut für Allgemeine Mikrobiologie, University of Bern, Baltzerstrasse 4, CH-3012 Bern, Switzerland. response is detectable as early as 5 days after infection, that this response is directed primarily against flagellar proteins and, quite unexpectedly, that uninfected mice exhibit considerable levels of high-affinity antibodies to at least two internal proteins of trypanosomes.

\section{MATERIALS AND METHODS}

\section{Trypanosomes and cell culture}

Trypanosoma brucei rhodesiense clone Lou'Tat 1 was used for infection experiments (De Gee et al. 1988). Procyclic T. b. brucei EATRO 427 and mouse 3T6 fibroblasts were grown as described earlier (Hemphill et al. 1992).

\section{Mice}

C57BL/6 mice were obtained either from the animal facility, University of Wisconsin, or from the Institut für Labortierkunde, University of Zürich, Switzerland; $\mathrm{C} 3 \mathrm{He} / \mathrm{B}$ mice were from BRL Ltd, Füllinsdorf, Switzerland or from the animal facility, University of Wisconsin; NOD mice were from the Institute of Pathology, University of Bern; germfree DBA/1 mice were from CIBA-Geigy, Basel, Switzerland; and outbred ICR mice were from the Institute für Labortierkunde, University of Zürich, Switzerland.

\section{Antibodies and immunodetection}

Antibody purification, immunoblotting and immunofluorescence were done exactly as described 


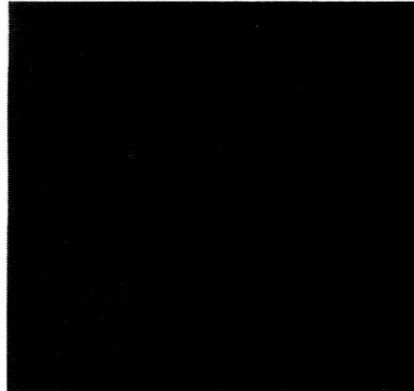

A

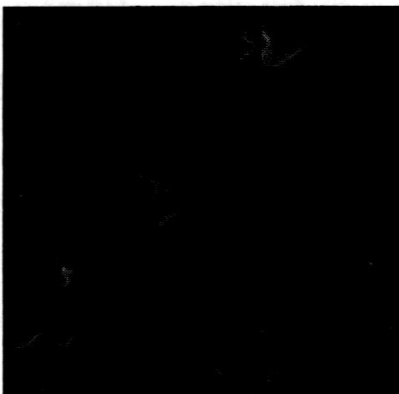

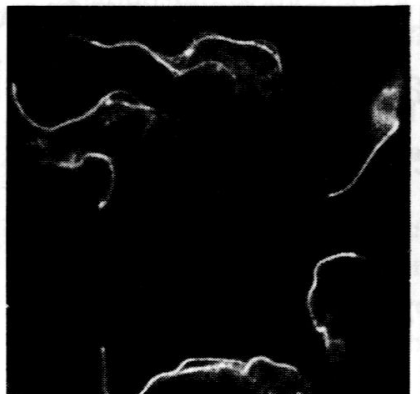

C

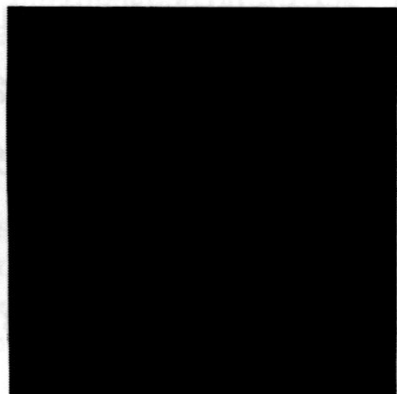

D

Fig. 1. Immunofluorescence staining of trypanosomes with sera taken at different times of infection.

Formaldehyde/methanol fixed procyclic trypanosomes were stained with serum from C57BL/6 mice taken before infection (A), or at days 5 (B) and 7 (C) after infection with Trypanosoma brucei rhodesiense LouTat 1. (D) Control staining with FITC-conjugated second antibody alone.

before (Hemphill et al. 1992; Müller et al. 1992). Sera from uninfected mice were used at a dilution of $1: 10$ for immunofluorescence and $1: 100$ for immunoblotting. Alkaline-phosphatase conjugated goat anti-mouse antibodies were from Promega (Madison, WI, USA) and were used at a dilution of 1:7500. FITC-conjugated rabbit anti-rat antibodies were from Dakopatts (Kopenhagen, Denmark) and were used at a dilution of $1: 100$.

\section{Preparation of antigens for Western blotting}

Recombinant I/6, MARP1 and paraflagellar rod protein were expressed in $E$. coli lysogenic for the corresponding gt11 expression phages as already described (Vogel et al. 1988). Trypanosomal cytoskeletons (Hemphill, Seebeck \& Lawson, 1991), cytoskeletal heat-resistant high-molecular weight proteins (Hemphill et al. 1992) and trypanosomal VSGs ('Theodos \& Mansfield, 1990) were prepared as described.

\section{RESULTS}

Time-course of appearance of anti-trypanosome antibodies after infection

Antigens against which an immune response of the host was mounted very early in infection were analysed by studying the trypanosome-specific antibody response during the early phase of an infection. C57BL $/ 6$ mice were infected i.p. with $T . b$.rhodesiense LouTat 1. Five animals were sacrificed at each of the times indicated in Fig. 1, and their pooled serum was analysed by immunofluorescence. Procyclic trypanosomes were used as targets in order to detect specifically the immune response to non-VSG antigens. The micrographs shown in Fig. 1 demonstrate that weak, but specific fluorescence became discernible at 5 days after infection (Fig. 1 B). A much stronger signal was observed at around day 7 (Fig. 1 C). Fluorescence at this stage was conspicuously concentrated along the flagellum. This observation is in close agreement with what has been seen in trypanosome infections of bovines (Müller et al., unpublished data), where serum taken early after infection also preferentially stained the flagellum.

\section{Trypanosomal proteins are recognized by antibodies of uninfected hosts}

The sera used above for immunofluorescence were further analysed for their ability to interact with a number of defined trypanosomal proteins. The proteins used in this assay were $\mathrm{I} / 6$ (Detmer $\&$ Imboden, unpublished observations) and MARP1 (Schneider, Hemphill \& Seebeck, 1988; Hemphill et al. 1992), which are both microtubule-associated proteins; GM6, a high molecular weight repetitive protein from the flagellar region (Müller et al. 1992); paraflagellar rod protein (Schlaeppi, Deflorin \& Seebeck, 1989); a heat-resistant high molecular weight protein fraction of microtubule-associated proteins; whole cytoskeletons of T.b.brucei; and purified VSG from the infecting strain LouTat 1. Sera from different time-points of infection $(0,5$ and $7 \mathrm{~d}$ ) were then used for immunostaining (Fig. 2A, B and $\mathrm{C}$, respectively). An unexpected result was obtained when immunostaining was done with preinfection serum (Fig. 2A). Two of the trypanosomal proteins included in the assay, I/6 and MARP1, produced strong immunostaining with this serum. A strong reaction was seen with the recombinant $\mathrm{I} / 6$ (Lane 1), while a weaker, but distinct, reaction was observed with the recombinant MARP1 (Lane 2), as well as with the heat-resistant high molecular weight fraction and the whole cytoskeleton (high molecular weight smear in Lanes 6 and 7), both of which contain the MARP1 protein (Hemphill et al. 1992). In contrast, no reaction of pre-infection serum was observed with either GM6 (Lane 3) or PFR (Lane $4)$, nor with the $\beta$-galactosidase control (Lane 5). This observation suggests that only a very limited set 


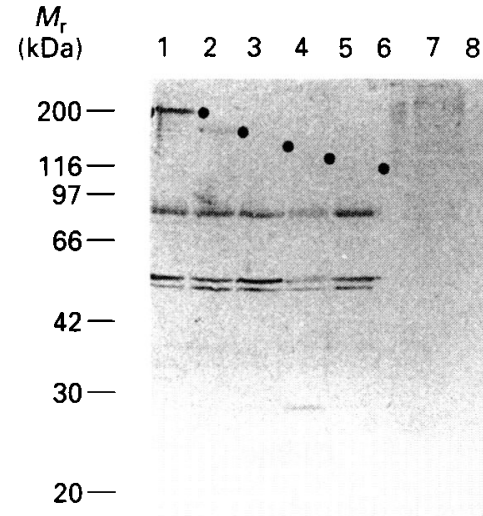

A

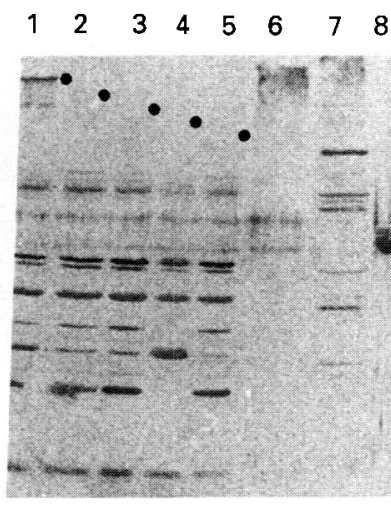

B

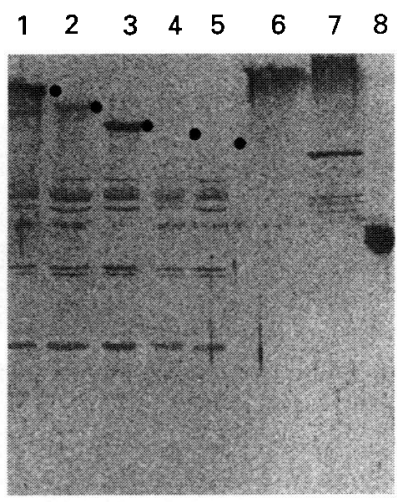

C

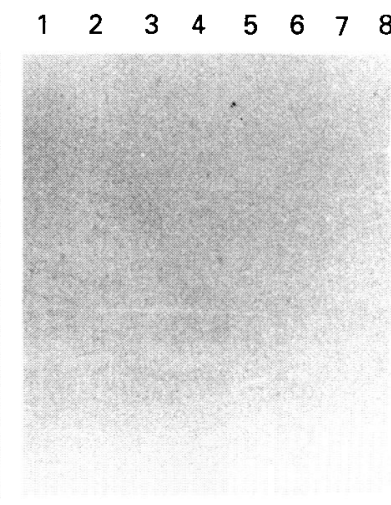

D

Fig. 2. Immunostaining of trypanosomal proteins with sera taken at different times after infection. E. coli lysates containing recombinant trypanosomal proteins (as $\beta$-galactosidase fusion proteins; Lanes $1-5$ ), subcellular fractions of trypanosomes (Lanes 6 and 7), and purified VSG LouTat 1.0 (Lane 8) were analysed by immunoblotting with serum from C57BL/6 mice taken before infection (A), or at days $5(\mathrm{~B})$ and 7 (C) after infection. Lane 1: protein I/6; Lane 2: MARP1; Lane 3: GM6; Lane 4: paraflagellar rod protein; Lane 5: $\beta$-galactosidase control; Lane 6 : heat-stable high molecular weight protein fraction of trypanosomes; Lane $7:$ trypanosome cytoskeletons; Lane 8 : VSG LouTat 1.0. (D) Control staining with phosphatase-conjugated second antibody alone. Dots in (A-C) indicate the position of the recombinant trypanosome proteins. All other bands visible are $E$. coli proteins.

of trypanosomal proteins is recognized by antibodies from uninfected hosts. In addition to the trypanosomal proteins, a small number of bacterial proteins were recognized by pre-infection serum in Lanes $1-5$, which contained $E$. coli lysates. The pattern of immunostaining produced by serum taken at 5 days post-infection testifies for the strong polyclonal stimulation of antibody production (Fig. 2 B). The immune response to the heat-resistant high molecular weight fraction (Lane 6) and the cytoskeleton fraction (Lane 7) was increased compared to the preinfection level. Also at this time-point, antibodies against the VSG LouTat 1 became discernible (Lane 8 ). Similarly, the immune response to $E$. coli proteins present in the bacterial lysates (Lanes 1-5) also became much stronger. A still higher level of antibody response was obtained with serum taken at day 7 (Fig. 2C), in agreement with the immunofluorescence data shown in Fig. 1. The 7-day serum reacted well with trypanosomal antigens $\mathrm{I} / 6$, MARP1 and GM6, the heat-resistant high molecular weight fraction, the cytoskeleton and VSG LouTat 1. In contrast, no antibody response was seen to bacterial $\beta$-galactosidase (Lane 5), nor to the paraflagellar rod protein of trypanosomes (Lane 4 ). This latter protein is very poorly immunogenic during trypanosomal infections, and no antibodies against it are produced even late in infection or when an infection in bovines is stopped by Berenil treatment (Müller, unpublished results).

\section{Antigen-recognition by different uninfected mouse strains}

The results presented in Fig. $2 \mathrm{~A}$ indicate that the serum of uninfected C57BL/6 mice recognized individual proteins from trypanosomes. In order to corroborate these findings, different inbred mouse strains, as well as outbred mice, were similarly analysed. Sera from 5 uninfected animals of each breed were pooled and analysed for their immunostaining patterns with the same array of trypanosomal proteins as used in Fig. 2. The breeds used in these experiments were the relatively trypano-resistant $\mathrm{C} 57 \mathrm{BL} / 6$, the highly trypanosusceptible $\mathrm{C} 3 \mathrm{HeB} / \mathrm{FeJ}$, non-obese diabetic (NOD) mice which exhibit enhanced levels of autoimmunity, and an outbred strain of white mice, ICR. Results shown in Fig. 3 demonstrate that sera from all strains produced very similar staining patterns for the trypanosomal proteins. Protein I/6 (Lanes 1) was strongly recognized by all. MARP1 was also detected by all sera, though at a lower level than I/6 (Lanes $2)$. The serum of the autoimmune NOD mice reproducibly yielded a stronger response to $I / 6$ and to the heat-resistant high molecular weight fraction than did the other sera (compare Lanes 1 and Lanes 6 of all panels). The similar staining pattern produced by all 4 sera with the trypanosomal proteins is in contrast to the obvious differences which are apparent in their reactivity toward the bacterial proteins present in the $E$. coli lysates applied in Lanes $1-5$.

These results confirm the initial observations obtained with C57BL/6 mice (Fig. 2) and indicate that serum from uninfected mice does in fact contain antibodies which are capable of reacting specifically with distinct trypanosomal proteins. In order to investigate if such antibodies might have arisen during earlier, subclinical infections of the animals with organisms expressing cross-reactive antigens, serum was obtained from germ-free DBA mice. A 


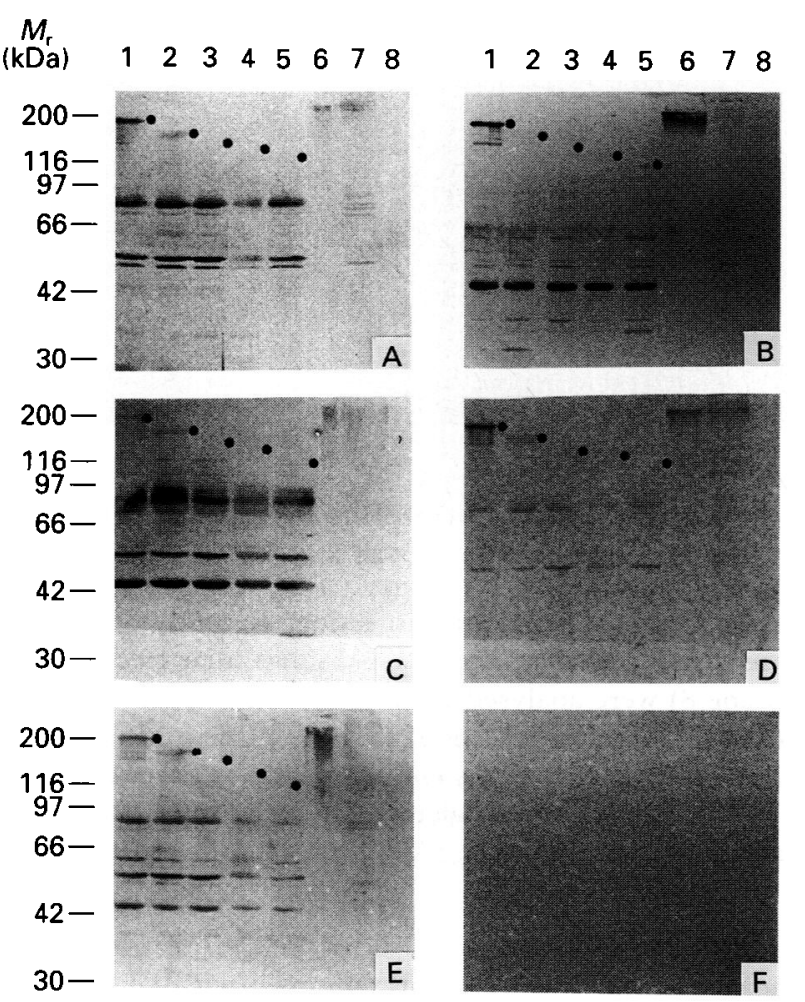

Fig. 3. The same set of trypanosomal proteins as used in Fig. 2 were immunostained with sera from different strains of uninfected mice. (A) C57BL/6; (B) NOD; (C) $\mathrm{C} 3 \mathrm{HeB} / \mathrm{FeJ}$; (D) DBA $/ 1$ (germ free); (E) ICR; (F) control. $\mathrm{C} 57 \mathrm{BL} / 6, \mathrm{C} 3 \mathrm{HeB} / \mathrm{FeJ}$ and $\mathrm{NOD}$ are inbred strains, ICR are outbred mice and DBA/1 were germfree animals. The control panel represents staining with phosphatase-conjugated second antibody alone. Dots indicate the position of the recombinant trypanosome proteins. All other bands visible are E. coli proteins.

comparison of the staining pattern produced by this serum with the patterns obtained from normally reared mice shows that this serum reacted with the trypanosomal proteins to a similar extent as did the sera from normal animals (Fig. 3). As expected, serum from germ-free animals produced a much weaker reaction to $E$. coli proteins (Lanes 1-5).

\section{Trypanosome-specific pre-infection antibodies are of IgM and IgG type}

Two parallel filters containing the standard array of proteins (see Fig. 2) were immunostained with serum from uninfected $\mathrm{C} 57 \mathrm{BL} / 6$ mice and were developed with antibodies specific for $\mathrm{IgG}$ and $\mathrm{IgM}$, respectively. Fig. 4 demonstrates that the mouse antibodies which reacted with proteins $I / 6$ and MARP1 (Lanes 1 and 2), with the heat-resistant high molecular weight fraction and with the cytoskeletal proteins (Lanes 6 and 7) were both of the IgM and IgG class. A very weak reaction to $E$. coli $\beta$-galactosidase was seen with $\operatorname{IgG}$, but not with IgM antibodies (Lanes 5 in Fig. $4 \mathrm{~A}$ and $\mathrm{B}$ ). The same weak reactivity was also seen with the $\beta$-galactosidase fusion proteins

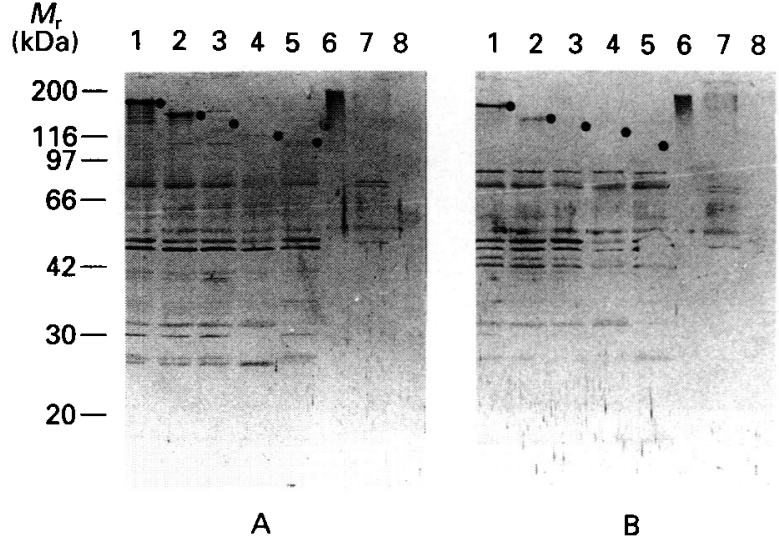

Fig. 4. Sets of the trypanosomal proteins as used in Figs 1 and 2 were stained with serum from uninfected C57BL/ 6 mice. Blots were then stained with an isotypespecific second rabbit anti-mouse antibody and were finally developed with phosphatase-conjugated antirabbit antibody. (A) IgG-specific staining; (B) IgMspecific staining. Dots indicate the position of the recombinant trypanosome proteins. All other bands visible are $E$. coli proteins.

with GM6 (Lane 3) and paraflagellar rod proteins (Lane 4). The comparison of antibody class and specificity presented in Fig. 4 indicates that trypanosome-reactive antibodies from uninfected animals were both of the IgM and the IgG class.

\section{Native antibodies to $I / 6$ and MARP1 are antigen specific}

The results presented so far have established that whole serum from uninfected animals contains antibodies which recognize the trypanosomal proteins $I / 6$ and MARP1. In a next step, these antibodies were affinity-purified from whole serum using $\beta$-galactosidase fusion proteins of $\mathrm{I} / 6$ and MARP1, respectively. As controls, hyperimmune sera raised against these same fusion proteins were processed in parallel. The specificity of the affinity-purified antibodies was then established by immunostaining filters which contained different trypanosomal extracts. Fig. 5 A and $\mathrm{B}$ show the comparative analysis of antibodies affinity-purified from hyperimmune and uninfected serum, respectively. Antibodies from both sources behaved very similarly. A strong reaction was seen with a lysate from an $E$. coli strain containing the $\beta$ galactosidase/I/6 fusion protein (Lane 1) while no reaction was detected with a control lysate containing $\beta$-galactosidase alone (Lane 2). When probed against trypanosomal cell lysates, no reaction was detected with the heat-resistant high molecular weight fraction (Lane 3), while characteristic biphasic staining (a high molecular weight component and a distinct double band at $32 \mathrm{kDa}$ ) was observed with the cytoskeletal lysate (Lane 4 ). This biphasic staining 

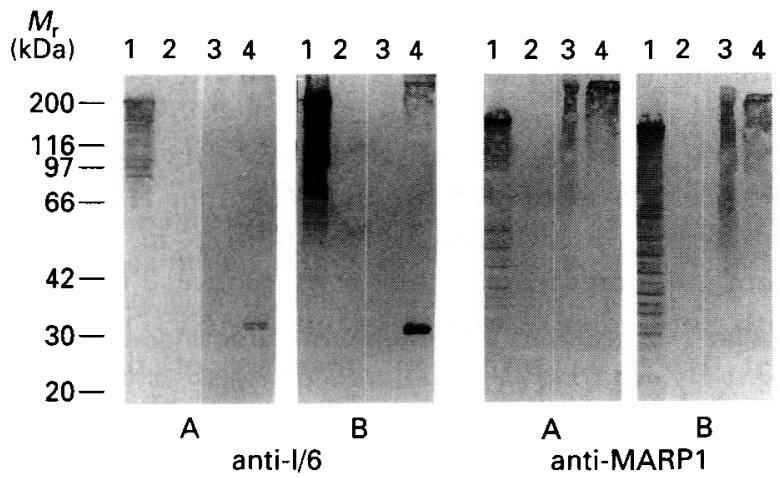

Fig. 5. Affinity purification of antibodies against trypanosomal proteins $\mathrm{I} / 6$ and MARP1 from the serum of uninfected mice. Specific antibodies were affinity purified either from uninfected mouse serum or from hyperimmune rat serum using the corresponding recombinant proteins as affinity matrices. The affinitypurified antibodies were used to stain the following immunoblots: Lanes $1: E$. coli lysate expressing the respective recombinant protein, Lanes $2: E$. coli lysate expressing wild type $\beta$-galactosidase; Lanes 3 : heatresistant high molecular weight proteins of trypanosomes; Lanes 4: trypanosome cytoskeletons. (A) Antibody purified from hyperimmune serum; (B) antibody purified from serum of uninfected C57BL $/ 6$.

pattern is typical for $I / 6$, and antibody affinitypurified on either the high molecular weight or the low molecular weight component visible on the gel, always recognized both components upon reprobing (Detmer et al. unpublished observations). Similarly, affinity-purified antibody against MARP1 specifically recognized the $\beta$-galactosidase/MARP1 fusion protein (Lanes 1), but not $\beta$-galactosidase alone (Lanes 2). On the trypanosomal lysates, this antibody recognized the heat-resistant high molecular weight fraction (Lanes 3) as well as high molecular weight components of the cytoskeleton (Lanes 4). Comparison of panels A and B of the MARP1 section again demonstrates that affinitypurified antibody from hyperimmune serum and from non-infected serum behave very similarly. In conjunction, the results presented in Fig. $5 \mathrm{dem}-$ onstrate that the serum of uninfected animals contains antibodies which are specific for individual trypanosome proteins, that these antibodies are of sufficiently high affinity that they can be purified by standard affinity procedures and, thirdly, that these antibodies behave very similarly to the corresponding hyperimmune antibodies.

The affinity-purified antibodies were also used to investigate whether they could have arisen due to an immune response to components of the mouse feed. Gut contents from all mouse strains tested in Fig. 3 were analysed by immunoblot, using affinitypurified antibodies against I/6 and MARP1. No reaction was found with any of these gut contents (results not shown).

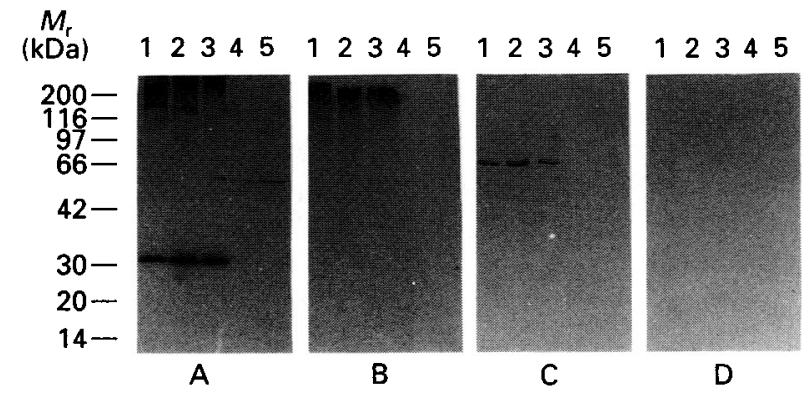

Fig. 6. Antibodies against proteins I/6, MARP1 and paraflagellar rod protein were affinity purified from hyperimmune rat sera and then used to stain immunoblots of trypanosomal and mouse subcellular fractions. Lanes 1: whole cell extract from bloodstream forms of Trypanosoma brucei; Lanes 2: whole cell extract of procyclic $T . b$. bruce $i$; Lanes 3 : trypanosome cytoskeletons; Lanes 4: whole cell extract of mouse $3 \mathrm{~T} 6$ cells; Lanes 5: cytoskeletons of mouse 3T6 cells.

(A) Anti-I/6 antibody; (B) anti-MARP1 antibody; (C) anti-PFR antibody; (D) control incubation with phosphatase-conjugated second antibody.

\section{Antibodies against I/ 6 from trypanosomes detect a $60 \mathrm{kDa}$ mouse protein}

The results obtained so far suggested that the trypanosome-specific antibodies from uninfected animals might represent anti-self antibodies. Antibodies against trypanosomal I/6 protein, MARP1 and paraflagellar rod protein were affinity-purified from hyperimmune serum from rats immunized with the corresponding $\beta$-galactosidase fusion proteins. The affinity-purified antibodies were then used for staining filters which contained cell extracts from trypanosomes and mouse 3T6 cells. Fig. 6A shows the staining of such a filter with $\mathrm{I} / 6$ antibody. In lysates of whole bloodstream trypanosomes, whole procyclic trypanosomes or trypanosome cytoskeletons (Lanes 1-3, respectively), the antibody displayed the typical biphasic staining pattern, recognizing a high molecular weight smear and a distinct band at $32 \mathrm{kDa}$. This latter band often migrated as a doublet (e.g. see Fig. 5, and unpublished observations). In lysates of whole mouse 3 T6 cells (Lane 4 ) or of 3 T6 cytoskeletons (Lane 5), the $I / 6$ antibody recognized a distinct band at $60 \mathrm{kDa}$. In contrast, antibodies against MARP1 and paraflagellar rod protein only recognized their appropriate target proteins in trypanosome cell lysates (Lanes 1-3, Fig. $5 \mathrm{~B}$ and C), but did not react with proteins in the mouse cell lysates (Lanes 4 and 5).

\section{The murine protein corresponding to trypanosomal I/6 may be a cytoskeletal protein}

The mouse cell protein which is reactive with antibody against trypanosomal I/6 was localized in the cytoskeleton as defined by the extraction procedure used (Fig. 6A, Lane 6). Affinity-purified 


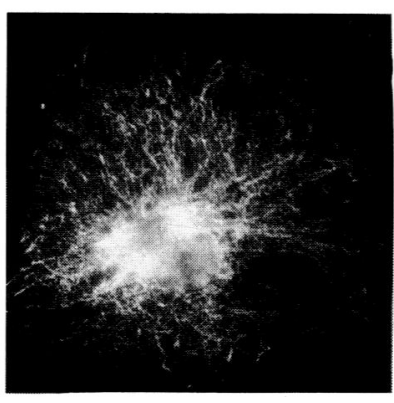

A

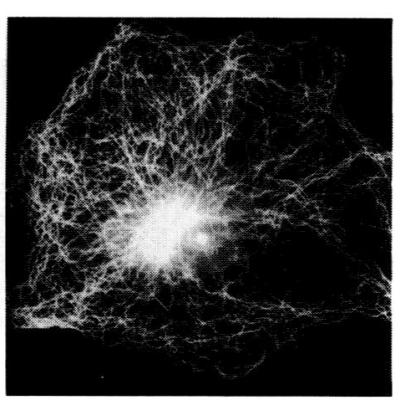

B
Fig. 7. 3T6 fibroblasts were fixed with methanol/acetone and double-stained with rat antibody against protein $I / 6$ and with rabbit antibody against tubulin. Localization of the corresponding proteins was then visualized by staining with FITC-conjugated anti-rat and Texas-Red conjugated anti-rabbit antibodies. The same cell is shown stained by anti- $\mathrm{I} / 6$ antibody (A) and by anti-tubulin antibody (B).

antibody was used to localize this protein by immunofluorescence in 3T6 cells. Cells were grown on cover-slips and were detergent extracted in situ. The resulting cytoskeletons were fixed and double label immunofluorescence was performed to simultaneously locate I/6 and microtubules (Fig. 7). The staining pattern obtained with the $I / 6$ antibody suggested that the corresponding protein was located on a filament system of the cytoskeleton (A), but that this system did not correspond to the microtubules (B). While immunofluorescence did not allow unambiguous identification of the cytoskeletal structure of which the I/ 6 corresponding protein was a part, it independently confirmed the results obtained above and established that the murine $I / 6$ homologue is a fairly major component of the murine cytoskeleton.

\section{DISCUSSION}

This study demonstrates that serum from uninfected mice contains antibodies against at least two different trypanosomal proteins, I/6 and MARP1. These native antibodies are of both the IgM and the IgG class, they are of sufficiently high affinity to enable their isolation by standard affinity purification procedures, and their staining pattern on trypanosome cell lysates is very similar to that obtained with hyperimmune antibodies against both proteins. They were found in all mouse strains tested, suggesting that their presence correlates neither with trypanosome resistance nor susceptibility, nor with a particular genotype or MHC haplotype. Furthermore, the observation that the same populations of antibodies are also present in germ-free animals indicates that they do not originate from previous infections of the animals with other pathogens. Nor is it likely that they are due to an immune response to components of the feed, since analyses of gut contents for cross-reacting components were nega- tive. In addition, animals were obtained from different breeders and were fed on different commercial chows.

Both I/6 and MARP1 are cytoskeletal, microtubule-associated proteins (Detmer et al. unpublished observations; Hemphill et al. 1992). Immunofluorescence analysis of mouse 3T6 cells with antibodies against $\mathrm{I} / 6$ and MARP1 revealed that $\mathrm{I} / 6$ is a prominent member of the $3 \mathrm{~T} 6$ cytoskeleton with a molecular weight of $60 \mathrm{kDa}$. Based on these observations, antibodies against $\mathrm{I} / 6$ appear to be natural anti-self antibodies. Antibodies against MARP1 may well be also of the anti-self type. The absence of immunostaining of 3T6 cells with antibody against MARP1 may indicate that this fibroblast line does not express the corresponding protein and suggests that its expression may be tissue- or cell-type specific. No native antibody response was detected against other trypanosomal proteins such as GM6 or paraflagellar rod protein. The results presented in this study have demonstrated that native antibodies against $I / 6$ and MARP1 are present in different mouse strains, as well as in outbred mice. However, similar populations of antibodies have recently also been found in other species of uninfected hosts such as human or bovine (Müller et al. unpublished observations), and variant-specific antibodies against several VSGs have recently been identified in sera from uninfected hosts (Müller et al. manuscript submitted). Our observations indicate that the presence of native antibodies against trypanosomes is a common phenomenon in mammalian hosts. Their potential role in the early phases of host/parasite interaction, as well as their possible interference with serological diagnosis of trypanosomiasis, remain to be explored.

We are grateful to Yvonne Schlatter for technical support, to Peter Müller of the Institute for Experimental Tumor Research, University of Bern, for Texas-Red conjugated antibodies, to André Schneider, Department of Molecular Parasitology, UCSF, San Francisco for isotype-specific antibodies, and to Isabel Roditi and Andrew Hemphill for many stimulating discussions. This study was supported by Swiss National Science Foundation grants $\mathrm{Nr} 31$ 30870.91 and $31-28574.90$ and a grant of the Stanley Thomas Johnson Foundation (to N.M. and T.S.) and an NIH grant (to J.M.M.).

\section{REFERENCES}

BANCROFT, G. J. \& ASKONAS, B. A. (1985). Immunobiology of African trypanosomiasis in laboratory rodents. In Immunology and Pathogenesis of Trypanosomiasis (ed. Tizard, I.), pp. 75-101. Boca Raton, Florida: CRC Press.

BURgeSs, D. E. \& JERRELls, T. (1985). Molecular identity and location of invariant antigens on Trypanosoma brucei rhodesiense defined with monoclonal antibodies reactive with sera from trypanosomiasis patients.

Infection and Immunity 50, 893-9. 
Cross, G. A. M. (1990). Cellular and genetic aspects of antigenic variation in trypanosomes. Annual Review of Immunology 8, 83-116.

DE GEE, A. L. W., LEVINE, R. F. \& MANSFIELD, J. M. (1988). Genetics of resistance to the African trypanosomes. VI. Heredity of resistance and variable glycoprotein specific immune responses. F. Immunol. 140, 283-8.

HEMPHILL, A., AFFOLTER, M. \& SEEBECK, T. (1992). A novel microtubule-binding motif identified in a high molecular weight microtubule-associated protein from Trypanosoma brucei. Journal of Cell Biology 117, 95-103.

HEMPHILl, A., SEEBECK, T. \& LAWSON, D. (1991). The Trypanosoma brucei cytoskeleton: ultrastructure and localization of microtubule-associated and spectrinlike proteins using quick-freeze, deep-etch immunogold electron microscopy. Fournal of Structural Biology 107, 211-20.

MORRISON, W. I., MURRAY, M. \& AKOL, G. W. O. (1985). Immune responses of cattle to African trypanosomes. In Immunology and Pathogenesis of Trypanosomiasis (ed. Tizard, I.), pp. 103-31. Boca Raton, Florida: CRC Press.

MÜlleR, N., hempHill, A., imboden, M., DUVAllet, G. DWINGER, R. H. \& SEEBECK, T. (1992). Identification and characterization of two repetitive non-variable antigens from African trypanosomes which are recognized early during infection. Parasitology 104, 111-19.
SCHLAEPPI, K., DEFLORIN, J. \& SEEBECK, T. (1989). The major component of the paraflagellar rod of Trypanosoma bruce $i$ is a helical protein that is encoded by two identical, tandemly linked genes. Fournal of Cell Biology 109, 1695-709.

SCHNEIDER, A., HEMPHILL, A. \& SEEBECK, T. (1988). Large microtubule-associated protein of $T$. brucei has tandemly repeated, near-identical sequences. Science 241, 459-62.

SHAPIRo, s. z. \& MURRAY, M. (1982). African trypanosome antigens recognized during the course of infection in $\mathrm{N}$ 'dama and Zebu cattle. Infection and Immunity 35, 410-16.

SHAPIRO, s. z. \& PEARSON, T. w. (1986). African trypanosomiasis: antigens and host-parasite interactions. In Parasite Antigens (ed. Pearson, T. W.), pp. 215-274. New York: Marcel Dekker. THEODOS, C. M. \& MANSFIELD, J. M. (1990). Regulation of $B$ cell responses to the variant surface glycoprotein molecule in trypanosomiasis. II. Down-regulation of idiotype expression is associated with the appearance of lymphocytes expressing antiidiotypic receptors. Fournal of Immunology 144, 4022-9.

VOGEL, M., MÜLLER, N., GOTTSTEIN, B. \& SEEBECK, T. (1988). Production of recombinant antigen of Echinococcus multilocularis with high immunodiagnostic sensitivity and specificity. Molecular and Biochemical Parasitology 31, 117-26. 